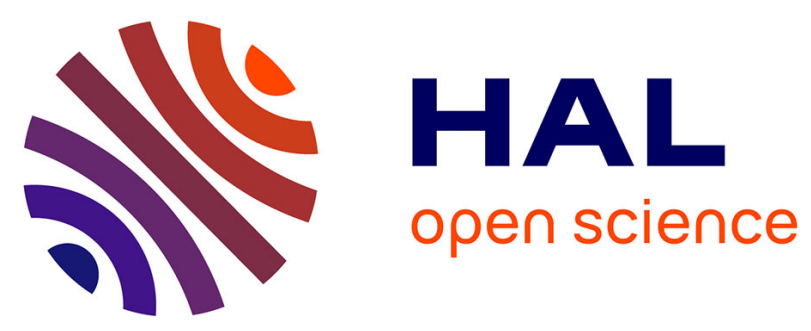

\title{
STRUCTURAL STABILITY OF TRANSITION AND NOBLE METAL CLUSTERS
}

\author{
J. Gaspard, C. Hodges, M. Gordon
}

\section{To cite this version:}

J. Gaspard, C. Hodges, M. Gordon. STRUCTURAL STABILITY OF TRANSITION AND NOBLE METAL CLUSTERS. Journal de Physique Colloques, 1977, 38 (C2), pp.C2-63-C2-67. 10.1051/jphyscol:1977213 . jpa-00217052

\section{HAL Id: jpa-00217052 https://hal.science/jpa-00217052}

Submitted on 1 Jan 1977

HAL is a multi-disciplinary open access archive for the deposit and dissemination of scientific research documents, whether they are published or not. The documents may come from teaching and research institutions in France or abroad, or from public or private research centers.
L'archive ouverte pluridisciplinaire HAL, est destinée au dépôt et à la diffusion de documents scientifiques de niveau recherche, publiés ou non, émanant des établissements d'enseignement et de recherche français ou étrangers, des laboratoires publics ou privés. 


\title{
STRUCTURAL STABILITY OF TRANSITION AND NOBLE METAL CLUSTERS (*)
}

\author{
J. , P. GASPARD \\ Institut de Physique, Université de Liège, \\ B-4000 Sart Tilman, Belgium

\section{H. HODGES and M. B. GORDON} \\ C.N.R.S., Laboratoire de transition de phases, \\ B.P. 166, F-38042 Grenoble, France
}

\begin{abstract}
Résumé. - Nous avons étudié l'énergie de cohésion de petites particules de métaux de transition et de métaux nobles en utilisant un modèle de liaisons fortes pour les bandes $s, p$ et $d$. Des particules de forme cubique et icosaédrique de 13,55 et 147 atomes ont été considérées. Nous avons examiné tout particulièrement la stabilité relative des structures cubiques et icosaédriques. Il apparaît que ces dernières sont toujours plus stables dans cette gamme de dimensions, indépendamment du remplissage des bandes et des paramètres de liaisons fortes choisis. La stabilité est dominée par le nombre moyen de premiers voisins d'un atome, qui est plus grand pour l'icosaèdre. En conséquence, nos résultats sont qualitativement les mêmes que ceux obtenus pour un potentiel de paire bien que notre modèle soit plus général.

Abstract. - The cohesive energy of small transition and noble metal particles has been investigated using a tight binding model for the $s, p$ and $d$ bands. Clusters of cubic and icosahedral form with 13,55 and 147 atoms have been studied. Special attention has been paid to the relative stability of the cubic and icosahedral structures. The latter always appears to be more stable in this size range, irrespective of the filling of the bands, and the particular tight binding parameters chosen. The stability is dominated by the average atomic coordination number which is larger for the icosahedron. Thus our results are qualitatively similar to those obtained using simple pairwise potential model, although it must be emphasized that our model is more general than that.
\end{abstract}

1. Introduction. - The aim of this paper is to discuss the structural stability of small transition metal and noble metal clusters. A number of investigations [1] have shown the existence of stable clusters of rare gas atoms showing icosahedral form. Experiments suggest the existence of five fold symmetry axes for small metallic particles (usually $\mathrm{Au}$ ). Such unusual symmetry, not found in regular crystalline lattices, is believed to have been observed for metal particles deposited from the vapour phase on an alkali halide substrate [2], nucleated in the vapour phase [3], and also for clusters formed inside a metallic matrix [4]. Such metallic particles may perhaps be formed by germination from icosahedral microclusters of less than 100 atoms or other structures showing pentagonal form [5]. Local pentagonal arrangements of atoms may occur in amorphous bulk materials and give rise to the splitting of the second peak in the pair distribution function [6].

The stability of the icosahedral or pentagonal microclusters may be understood as a nearest neighbour bonding effect. Simple geometrical considerations show that, for example, the f.c.c.

$\left.{ }^{*}\right)$ Work partly supported by the Belgian ESIS program, and by A.T.P. Surface Contract $\mathrm{N}^{\circ} 2038$. nearest neighbour coordination polyhedron (12 surface atoms plus one central atom) may undergo a small distortion to a 13 atom icosahedron and thereby increase the coordination of each of its surface atoms from 5 to 6 . Thus on a simple attractive nearest neighbour bond model the icosahedron should be energetically favoured. The icosahedron with its five fold symmetry axes may serve as a nucleus for larger clusters of similar symmetry ; indeed there exists a series of clusters of perfect icosahedral form containing $13,55,147$ etc. atoms. For each of these numbers there is also a corresponding cluster counterpart based on the undistorted f.c.c. lattice.

The structure of the icosahedral clusters may be understood as a multiple twinning of slightly distorted f.c.c. tetrahedra [2], in such a way that the surface consists entirely of close packed [111] planes of atoms for which the surface energy is lowest [7]. On the other hand the f.c.c. cluster surface presents in part the less densely packed [200] planes, whose atoms are less highly coordinated leading to a higher surface energy. The stability of the icosahedral particles is thus essentially a surface effect and so seems to be a property shared by a lot of systems like rare gases, transition metals, noble metals... The elastic distortion energy 
of the icosahedral tetrahedra, which is initially very small but grows in proportion to the volume, will however dominate the surface energy in the limit of large particle size thus eventually destabilising the icosahedral form [7]. Similar considerations also apply to sequences germinated by other microclusters showing five fold symmetry e.g. the pentagonal bipyramid.

The arguments outlined above, based on the competition between the classical surface energy and volume elastic energy are certainly appropriate for sufficiently large particle size. Hoare and Pal [1] who made extensive investigations of clusters less than 100 atoms reached similar conclusions for particles interacting via pairwise Lennard Jones or Morse potentials, as would happen for the atoms of a rare gas. They found the 13 and 55 atom icosahedra to be the most stable of all the particle configurations they considered. For 13 atoms, the difference in icosahedral and f.c.c. cluster energies may be as much as $17 \%$.

The question is whether this remains true for elements other than rare gases, in particular transition and noble metals, where the pairwise force model is no longer appropriate. In these cases, the distribution of the electronic energy levels, especially those of the $d$ band, plays a central role in determing the cohesive energy. For the very small clusters one could imagine that specifically quantum mechanical effects, such as the filling of discrete electronic energy levels of large degeneracy (cf. the shell model of nuclear stability) or the inherent instability of a structure with a degenerate ground state (the Jahn Teller effect) might play some role in influencing the structural stability.

We should remark that at present even the most sophisticated quantum mechanical calculations may have difficulty in predicting ab initio the correct stable structure when the energy differences involved are small, as they often are. However we believe quite a lot may be learned from relatively unsophistical model calculations, provided one concentrates on investigating the factors influencing the choice of different structures.

2. Method. - Quantum mechanical calculations on small clusters of icosahedral symmetry have been performed by various authors [8, 9, 10$]$. Wheelock and Burton [9] who studied the cohesive energy of $\mathrm{Ni}$ clusters up to 13 atoms using the extended Hückel molecular orbital method, found relatively small energy differences between clusters of cubic and icosahedral symmetry. Indeed for 13 atoms the energy difference was less than $1 \%$.

We have reexamined this question within the framework of a tight binding description of the electronic structure. We take as our point of departure the following formula for the cohesive energy $E_{\mathrm{c}}$

$$
E_{\mathrm{c}}=\sum_{i}\left(E_{i}-E_{0}\right) d_{i}+E_{\mathrm{rep}}
$$

Here the sum is over the one electron eigenvalues, energy $E_{i}$, degeneracy $d_{i}$, and $E_{0}$ is the atomic energy level which is roughly at the centre of the band. The first term in (1) is an attractive contribution to the cohesive energy, which we have computed and shall discuss in terms of band broadening and hybridisation. The second term represents a repulsive interaction between the atoms which can be described phenomenologically by for instance a Born Mayer pair potential. We have not explicitly included this repulsive potential in our calculations, but for small clusters we do not believe it affects drastically the structural dependence of the cohesive energy, see $\S 3$. It would however become important for large cluster sizes, where the strain energy calculated by no contributes to the destabilization of the icosahedron.

We have chosen to describe the band broadening and hybridisation using the simplified tight binding model Hamiltonian due to Slater and Koster [11] with hopping parameters obtained by fitting to first principles band calculations for the bulk solid. We have used interpolation schemes for $\mathrm{Cu}$ [12] for the $3 d$ series and Pd [13] for the 4d series. Both include the $s-p$ bands in the tight binding representation. Our results were not found to be very sensitive to the particular set of parameters used. As the tight binding parameters must vary smoothly throughout the transition series it was possible to investigate qualitatively the variation of cohesion or cluster stability with electron/atom ratio within a rigid band model using a single set of parameters. Too much significance should not be attached to the absolute magnitude of our cohesive energies because of possible rescaling of the parameters from element to element.

In the calculations only nearest neighbour hopping parameters have been used, since with increasing distance they tend rapidly to zero [12]. In the icosahedron there are two nearest neighbour distances differing by $5 \%$. The same parameters have been used for both distances (and in the f.c.c. cluster) as the variation of hopping parameter with distance is not known to any certainty. It may be shown that by doing this one makes an error proportional to $\varepsilon^{2}$ in the equilibrium cohesive energy with $\varepsilon=0.05$. For small clusters (13, 55 atoms) this error is completely insignificant. Our procedure amounts to putting the average nearest neighbour bond length in the icosahedron equal to that in the f.c.c. cluster and bulk. The variation of bond length with cluster size and form remains to be investigated in detail.

In determining the cluster eigenvalues two different approaches have been used. The first involves an exact diagonalization of the tight binding Hamiltonian which is easy to carry out for the 13 atom clusters. The second approach uses an approximate 
diagonalization of the Hamiltonian based on a truncated continued fraction technique [14] which preserves the moments of the density of states. The accuracy of this method was checked by comparison with the results of exact diagonalization for the 13 atom cluster.

The calculations presented here are not selfconsistent. We have however made preliminary investigations of the effect of going to selfconsistency for the 13 atom clusters. This may be achieved in an approximate way by renormalising the atomic level of the central atom so as to ensure charge neutrality on each atom. The change in cohesive energy introduced by the self-consistent redistribution of electronic charge has been found always to be much smaller than the structural energy difference (cf. Fig. 2). In any case for Nickel and Copper our non self-consistent calculations are already very close to the charge neutrality condition.

3. Results and discussion. - In figure 1 we present the spectrum of eigenvalues for the 13 atom cubic and icosahedral clusters. For convenience the case of a pure unhybridised $d$ band is shown. Although the cubic spectrum has a larger extreme width, the effective width as defined by the second moment of the density of states is larger for the icosahedron. This may be shown to be a result of the larger nearest neighbour coordination of the icosahedron, since the second moment is proportional to the average number of bonds per atom $\bar{n}_{c}$. The higher degeneracy of the icosahedron levels is à consequence of its higher symmetry [15].

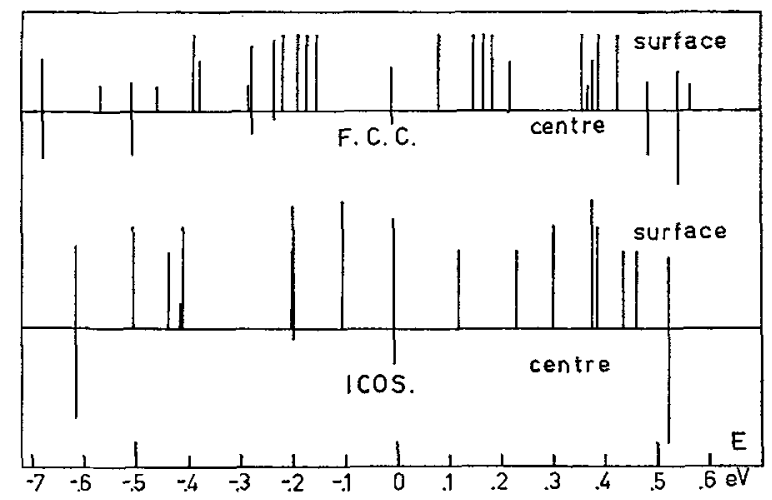

FIG. 1. - Cluster energy levels (13 atoms) for a pure d band, (a) icosahedral, (b) cubic. The $y$ axis represents the total squared amplitude of a degenerate set of eigenfunctions on the surface atoms or central atom (plotted upwards and downwards respectively).

In figure 2 the cohesive energies of the same 13 atom clusters are compared as a function of band filling using the Kleinmann $\mathrm{Cu}$ parameters. The icosahedral cluster appears to be more stable irrespective of electron atom ratio. This result remains true for the Pd parameters and for the 55 atom clusters. It is consequence of the larger

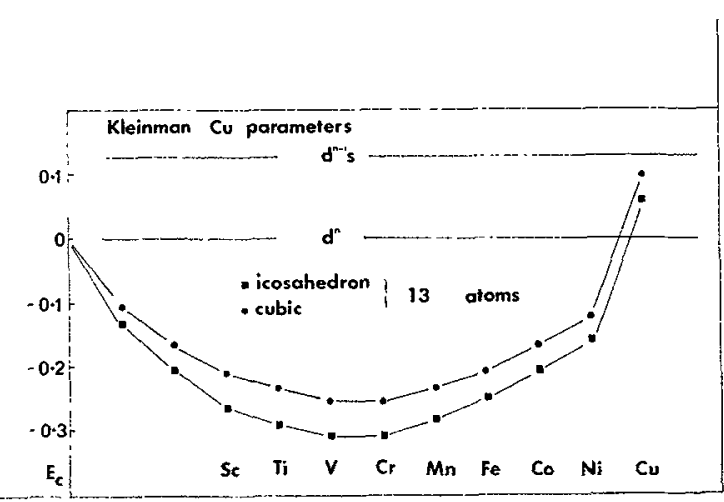

FIG. 2. - Comparison of the cohesive energy $E_{\mathrm{c}}$ in Ry/atom for 13 atoms calculated using the hybridised $d, s, p$ parameters of Kleinman. The energy is shown relative to atomic configurations $\mathrm{d}^{n}$ and $\mathrm{d}^{n-1} \mathrm{~s}$, the latter being more appropriate for the noble metals (the atomic d level is assumed unchanged in the cluster, but the $d \rightarrow s$ promotion energy has been deduced from the atomic spectroscopic data rather than the bulk tight binding parameters).

effective bandwidth for the icosahedron resulting from the higher coordination of its surface atoms. At the end of the transition series and the noble metals the energy difference is due in part to the s-d hybridisation which is again larger for the icosahedron. For these elements the Faulkner parameters give a smaller energy difference since the hybridisation is weaker.

If one approximates the density of states by a curve of fixed shape whose width is fitted in the second moment $\mu_{2}$, the band broadening energy must be proportional to $\sqrt{\mu_{2}}[16]$ i.e. to the square root of the average coordination number $\bar{n}_{\mathrm{c}}$. This relation, is illustrated by the numerical results shown in figure 3 for a half filled $\mathrm{d}$ band. The percentage attractive energy difference between cubic and icosahedral structures decreases with

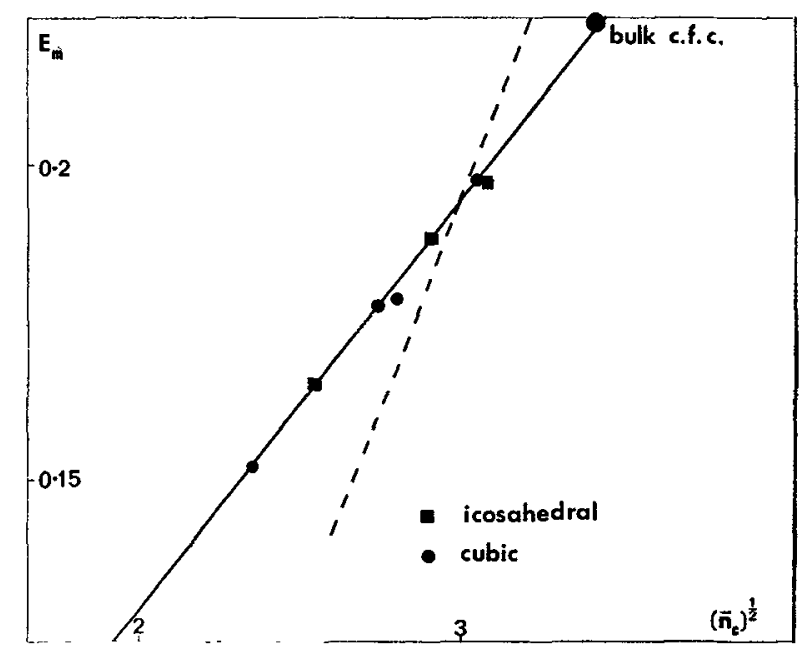

FIG. 3. - Variation of the midband cohesive energy $E_{m}$ in $\mathrm{Ry} /$ atom showing the proportionality to the root mean atomic coordination $\left(\bar{n}_{c}\right)^{1 / 2}$. The dashed line represents the type of variation which would be obtained from a short ranged pairwise potential $\left(E_{m} \propto \bar{n}_{\mathrm{c}}\right)$. The points are the results of exact calculations for a pure $d$ band on cubic and icosahedral clusters ranging from 13 to 201 atoms. 
increasing size owing to the decreasing surface to volume ratio. When this difference becomes less than the strain energy difference, the icosahedron will become metastable as has been discussed by no [7]. Let us remark that the bulk value of the f.c.c. crystal was found to lie nicely on the curve extrapolated to $\bar{n}_{\mathrm{c}}=12$.

Our results show that the detailed form of the density of states (energy spectrum), which is determined by the higher moments, has relatively little effect on the cohesive energy. Thus there is nothing to indicate that the position of the Fermi level with respect to special features in the density of states has very much influence on the relative stability of the two structures as it does in the theory of alloys for example ; the icosahedral small clusters are found to be the most stable irrespective of the $d$ band filling. The only case we found exhibiting anything analogous to the electron/atom stability regime encountered in alloys was that of a very idealized model, namely a single $s$ orbital per atom, where the cubic structure is more stable in a small range aroung electron/atom (half filled $s$ band). This stability occurs when a five fold degenerate icosahedron level is partially filled. A lowering of the symmetry to cubic, which splits this level into a 3 fold and 2 fold degenerate state, may then be energetically favourable as in the Jahn Teller effect. However the addition of $s-d$ and $s-p$ hybridisation is apparently enough to distroy this phenomenon. It would also be reduced by the extra Hund's rule stability (not included in our model) of the partially filled five fold level.

We should perhaps make some final remarks concerning the repulsive Born-Mayer potential which we have not included explicitly in our calculation. It is well known [17] that at equilibrium the repulsive contribution to the cohesive energy is small owing to the rapid fall-off of the Born-Mayer potential with distance. Thus although this contribution is larger for the icosahedron owing to the larger average coordination in that structure, we do not expect it to reverse the structural trend unless the attractive band broadening energy is very similar in the two clusters. One may be a little more quantitative by supposing the attractive and repulsive parts of the cohesive energy to scale exponentially with cluster size $R$, i.e.

$$
\begin{aligned}
& E_{\text {rep }}=A \exp \left[-p\left(R-R_{0}\right)\right] \text { and } \\
& E_{\text {att }}=-B \exp \left[-q\left(R-R_{0}\right)\right],[18]
\end{aligned}
$$

The repulsive exponent $p$ is generally reckoned to be about 3 times the attractive one $q[18]$. By minimizing (1) with respect to $R$, one finds that the cohesive energy at equilibrium varies as

$$
E_{\mathrm{eq}} \propto\left[B^{P} A^{-q}\right] \frac{1}{p-q}
$$

where $B$ and $A$ are the attractive and repulsive energies at a standard $R_{0}$, as in our model. The repulsive part $A$ is simply proportional to the mean coordination $\bar{n}_{\mathrm{c}}$ which is greater in the icosahedron by $17 \%$. Since however $p / q$ is roughly 3 , this would mean that the relative stability of the two clusters would change sign only if the attractive part $B$ differs by less than 5 or $6 \%$. Such is clearly not the case for the results presented in figure 2 , though we did find differences as small as $6 \%$ for parts of the transition series using the Faulkner Pd parameters.

In conclusion, we should emphasize that further progress in predicting the stability of specific structures is not possible without a reliable way of calculating tight binding parameters and their variation with bond length. Our investigation here has however shown that the mechanism of isocahedron stabilisation due to increasing atomic coordination remains in general valid within a tight binding model. We must also point out that the present work is restricted to considering relative structural stability for the very special cluster sizes of 13,55 , 147, etc. atoms. In between these numbers there are cubic clusters closely approaching the energetically favourable Curie-Wulff polyhedron and having no icosahedral counterparts. It is perfectly possible for an icosahedral microcluster of sufficiently small size to change to a cubic growth sequence on adding further atoms, and also possible that reverting to an icosahedral form becomes less and less probable as time goes on, because rearrangements of larger and larger numbers of atoms are involved. Thus it is clear that the existence of the type of five fold cluster observed in the electron microscope depends crucially on dynamic growth mechanisms which we have not considered here.

Acknowledgments. - We are grateful for guidance and discussions with $\mathrm{M}$. C. Desjonquères and F. Cyrot-Lackmann.

\section{References}

[1] Hoare, M. R., Pal, P., Adv. Phys. 24 (1975) 645. Farges, J., Raoult, B., Torchet, G., J. Chem. Phys. 59 (1973) 3454

[2] INo, S., J. Phys. Soc. Japan 21 (1966) 346.
[3] Solliard, C., Buffat, P., Faes, F., J. Cryst. Growth 32 (1976) 123.

[4] Fargues, D., Thesis, Univ. Paris VI (1976), unpublished.

[5] Burton, J. J., Cat. Rev.-Sci. Eng. 9 (1974) 209. 
[6] Dixmier, J., Bletry, J., Sadoc, J. F., J. Physique Colloq. 36 (1974) C2-65.

[7] Ino, S., J. Phys. Soc. Japan 27 (1969) 941.

[8] JULG, A., Bourg, M., J. Physique Colloq. 36 (1975) C2-31. FRIPIAT, J. G. et al., to be published.

[9] WHEELOCK, K. S., BURTON, J. J., to be published.

[10] Leleyter, M. and JoYes, P., J. Physique Lett. 36 (1976) L-303.

[11] Slater, J. C., Koster, G. F., Phys. Rev. 94 (1954) 1498.

[12] Sohn, K. S., Dempsey, D. G., Kleinman, L., Caruthers, E., Phys. Rev. B 13 (1976) 1515.

[13] FaulKner J., Phys. Rev. B 13 (1976) 2391.
[14] Gaspard, J. P., Cyrot-Lackmann, F., J. Phys. C 6 (1973) 3077:

Haydock, R., Heine, V., Kelly, M. J., J. Phys. C5 (1972) 3845 .

[15] Gaspard, J. P., A.I.P. Proc. Conf. on Structure and Excitations of Amorphous Solids, Williamsburg, 1976 (to be published).

[16] Ducastelle, F., Cyrot-lackmann, F., J. Phys. Chem. Solids 32 (1970) 285.

[17] MOTT, N. F., JONES, H., The theory of the properties of metals and alloys (Dover : New York, 1936) p. 144-145.

[18] Ducastelle, F., J. Physique 31 (1970) 1055. 